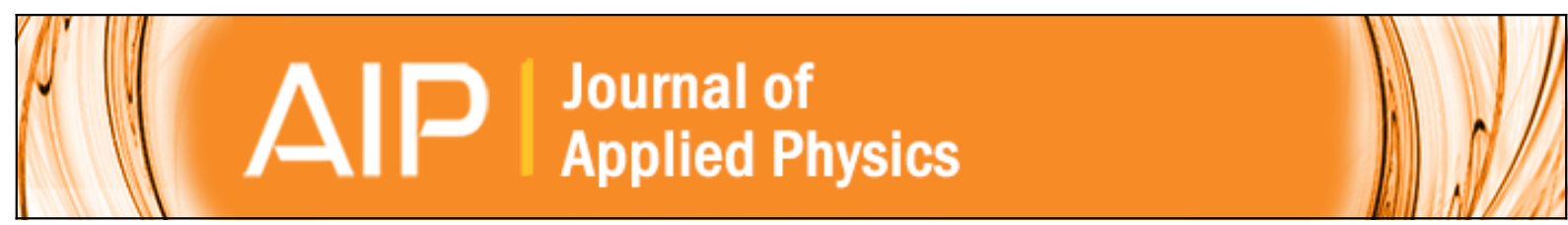

\title{
Room temperature ferromagnetism in liquid-phase pulsed laser ablation synthesized nanoparticles of nonmagnetic oxides
}

S. C. Singh, R. K. Kotnala, and R. Gopal

Citation: Journal of Applied Physics 118, 064305 (2015); doi: 10.1063/1.4928312

View online: http://dx.doi.org/10.1063/1.4928312

View Table of Contents: http://scitation.aip.org/content/aip/journal/jap/118/6?ver=pdfcov

Published by the AIP Publishing

\section{Articles you may be interested in}

Room temperature ferromagnetism in defective $\mathrm{TiO} 2$ nanoparticles: Role of surface and grain boundary oxygen vacancies

J. Appl. Phys. 114, 203906 (2013); 10.1063/1.4833562

Structural, optical, vibrational, and magnetic properties of sol-gel derived Ni doped $\mathrm{ZnO}$ nanoparticles

J. Appl. Phys. 114, 033912 (2013); 10.1063/1.4813868

Room temperature ferromagnetism in non-magnetic doped $\mathrm{TiO} 2$ nanoparticles

J. Appl. Phys. 113, 17B511 (2013); 10.1063/1.4795615

Enhanced indirect ferromagnetic $\mathrm{p}-\mathrm{d}$ exchange coupling of $\mathrm{Mn}$ in oxygen rich $\mathrm{ZnO}: \mathrm{Mn}$ nanoparticles synthesized by wet chemical method

J. Appl. Phys. 111, 033503 (2012); 10.1063/1.3679129

High temperature ferromagnetism and optical properties of Co doped $\mathrm{ZnO}$ nanoparticles

J. Appl. Phys. 108, 084322 (2010); 10.1063/1.3500380

\section{PIDEN}

\section{Instruments for Advanced Science}

Contact Hiden Analytical for further details: w www.HidenAnalytical.com E info@hiden.co.uk CLICK TO VIEW our product catalogue

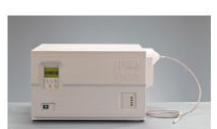

Gas Analysis

dynamic measurement of reaction gas streams catalysis and thermal analysis

molecular beam studies

' dosolved speciesprobes

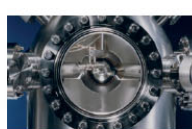

Surface Science

UHVTPD

SIMS end point detection in ion beam etch

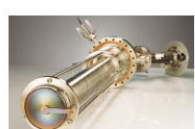

Plasma Diagnostics plasma source characterization etch and deposition process reaction , analysis of neutral and radical species

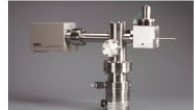

Vacuum Analysis partial pressure measurement and control of process gases reactive sputter process contro , vacuum diagnostics vacuum coating process monitoring 


\title{
Room temperature ferromagnetism in liquid-phase pulsed laser ablation synthesized nanoparticles of nonmagnetic oxides
}

\author{
S. C. Singh, ${ }^{1, a)}$ R. K. Kotnala, ${ }^{2}$ and R. Gopal ${ }^{1}$ \\ ${ }^{1}$ Laser Spectroscopy and Nanomaterials Lab, Department of Physics, University of Allahabad, \\ Allahabad-211002, India \\ ${ }^{2}$ Magnetic Standardization Division, National Physical Laboratory, K.S. Krishnan Road, \\ New Delhi, India
}

(Received 18 April 2015; accepted 12 June 2015; published online 13 August 2015)

\begin{abstract}
Intrinsic Room Temperature Ferromagnetism (RTF) has been observed in undoped/uncapped zinc oxide and titanium dioxide spherical nanoparticles (NPs) obtained by a purely green approach of liquid phase pulsed laser ablation of corresponding metal targets in pure water. Saturation magnetization values observed for zinc oxide (average size, $9 \pm 1.2 \mathrm{~nm}$ ) and titanium dioxide (average size, $4.4 \pm 0.3 \mathrm{~nm}$ ) NPs are 62.37 and $42.17 \mathrm{memu} / \mathrm{g}$, respectively, which are several orders of magnitude larger than those of previous reports. In contrast to the previous works, no postprocessing treatments or surface modification is required to induce ferromagnetism in the case of present communication. The most important result, related to the field of intrinsic ferromagnetism in nonmagnetic materials, is the observation of size dependent ferromagnetism. Degree of ferromagnetism in titanium dioxide increases with the increase in particle size, while it is reverse for zinc oxide. Surface and volume defects play significant roles for the origin of RTF in zinc oxide and titanium dioxide NPs, respectively. Single ionized oxygen and neutral zinc vacancies in zinc oxide and oxygen and neutral/ionized titanium vacancies in titanium dioxide are considered as predominant defect centres responsible for observed ferromagnetism. It is expected that origin of ferromagnetism is a consequence of exchange interactions between localized electron spin moments resulting from point defects. (C) 2015 AIP Publishing LLC.
\end{abstract}

[http://dx.doi.org/10.1063/1.4928312]

\section{INTRODUCTION}

Room temperature ferromagnetism (RTF) in diluted magnetic semiconductors (DMSs) that contain small amount of magnetic impurities are of potential interest recently, due to their expected spintronic applications of processing and memory unit fabrication at the same point for the construction of faster and cheaper computing and information devices. Small amount of transition metals (TMs) such as Mn, $\mathrm{Ni}, \mathrm{Co}$, etc., are mostly used as dopants of magnetic elements into semiconductor host to obtain room temperature $\mathrm{DMSs}^{1-4}$ at the early days of spintronic research. Zinc oxide is one of the best predicted promising host material for the development of room temperature DMSs, ${ }^{5}$ which is therefore exploited extensively for spintronic applications. Titanium dioxide is the best competitor of $\mathrm{ZnO}$ for photonic, electronic, and energy harvesting device fabrications, and it also deserves to be an excellent DMS host material. ${ }^{6,7}$ Not only transition metals but also doping of non-magnetic elements such as $\mathrm{C}^{8}$ and $\mathrm{Li}^{9,10}$ into diamagnetic zinc oxide or its capping by non-magnetic organic molecules such as thiol, amine, topo, etc., to modify electronic configuration of host materials, also induces RTF. ${ }^{11}$ Doping or capping of the host material may induce un-pairing of electrons, which may be the cause of ferromagnetism in $\mathrm{Li}$ and $\mathrm{C}$ doped and organic materials capped nonmagnetic oxides. ${ }^{9-11}$ The key

\footnotetext{
a) Author to whom correspondence should be addressed. Electronic mail: subhash.laserlab@gmail.com
}

challenges for such materials are to produce and preserve high value of saturation magnetization $\left(\mathrm{M}_{\mathrm{s}}\right)$ and retentivity $\left(\mathrm{M}_{\mathrm{r}}\right)$ at room and higher temperatures for their possible applications in spintronic device fabrications.

While TM doped DMSs were facing a lot of controversies about the existence of long range ferromagnetism with Curie temperature $\left(\mathrm{T}_{\mathrm{c}}\right)$ of $300 \mathrm{~K}$ or higher, observation of magnetism in undoped thin films of non-magnetic $\mathrm{HfO}_{2}$ arisen great challenge for the existing theories of magnetic materials. ${ }^{12}$ Origin of ferromagnetism from nonmagnetic nature of $\mathrm{Hf}^{+4}$ and $\mathrm{O}^{-2}$ ions of bulk diamagnetic hafnium dioxide made surprised to the scientific community and motivated theoretical and experimental investigations in the field of intrinsic ferromagnetism. It was suggested that magnetism in TM doped DMSs is the consequence of double exchange, ${ }^{13}$ while for nonmagnetic element doped DMSs, it may originate from the clustering and isolation of dopants ${ }^{14}$ or modification in the basic electronic configuration of the host. ${ }^{11}$ Ferromagnetism in undoped diamagnetic metal oxide nanomaterials mainly originates from the point defects created in the process of their quantum confinements, which therefore highly depends on the route of sample preparation. Nanomaterials produced by highly non-equilibrium processes such as pulsed laser ablation followed by plasma condensation in nanosecond and femtosecond time regimes have better chances of defect generation and hence modification in its local electronic configuration. First observation of RTF by Venkatesan et al. on $\mathrm{HfO}_{2}$ thin film, ${ }^{12}$ report of 
Hong et al. on undoped $\mathrm{TiO}_{2}, \mathrm{HfO}_{2}$, and $\mathrm{In}_{2} \mathrm{O}_{3}$ thin films, ${ }^{15}$ and the most recent work of Yi et al. on the thin films of $\mathrm{Li}$ doped $\mathrm{ZnO}^{10}$ are all based on the pulsed laser deposition (PLD), which is a non-equilibrium processing method. In the particular case of RTF in spherical undoped nanoparticles (NPs) of diamagnetic oxides, Sundaresan et al. annealed samples at $400-500{ }^{\circ} \mathrm{C}$ under $\mathrm{O}_{2}$ flow, ${ }^{16}$ while Garcia et al. ${ }^{11}$ used capping of zinc oxide NPs by organic molecules for alteration in their local electronic configuration to produce ferromagnetism. Similar to these, Zhou et al. used oxygen ion beam irradiation on a single crystalline $\mathrm{TiO}_{2}$ film to create ferromagnetism responsible for point defects. ${ }^{17}$ Any type of defect such as neutral/cation/anion deficiencies/excess that can alter outer cell electronic configuration to create unpaired electrons on their neighboring ions might induce para/ferromagnetism in nonmagnetic materials. Synthesis of NPs and their postprocessing treatments in these works ${ }^{11,16}$ involve several complex chemical processes, which therefore have the possibility of getting contaminated. Here, we present a simple, one step, purely green and physical approach, which is cheaper and simpler compared to PLD, and enable no chance of chemical contamination like chemical methods, to synthesize $\mathrm{ZnO}$ and $\mathrm{TiO}_{2}$ NPs with high order of RTF and Curie temperature. To the best of our knowledge, this is the first report on the RTF in the undoped, uncapped, assynthesized spherical NPs of diamagnetic semiconductor oxides.

\section{EXPERIMENTAL}

Nanoparticles of zinc oxide and titanium dioxide were synthesized by liquid phase pulsed laser ablation (LP-PLA, water as a liquid in this case) of corresponding metal targets. The details of synthesis procedure and sample characterizations are described in our previous reports. ${ }^{18}$ Briefly, high purity metal $\left(99.99 \%\right.$, Specpure ${ }^{\circledR}$, Johnson Mathey, UK) rod of $\mathrm{Zn}$ or $\mathrm{Ti}$, placed at the bottom of glass vessel containing high purity double distilled water (Merck, Inc.), was allowed to irradiate with focused output from pulsed Nd:YAG laser (Spectra Physics, QuantaRay, USA) operating at a $35 \mathrm{~mJ} /$ pulse energy, a $10 \mathrm{~Hz}$ repletion rate, and a $10 \mathrm{~ns}$ pulse width. The height of the water above the target surface, laser irradiance at the target, and the time of ablation were $30 \mathrm{~mm}$, $6 \times 10^{10} \mathrm{~W} / \mathrm{cm}^{2}$, and $60 \mathrm{~min}$ respectively for all the experiments. Violet colored highly stable colloidal solution of NPs and yellowish colored suspensions were obtained after water phase PLA of titanium and zinc rods, respectively. Particles were separated from solution by ultrahigh centrifugation $(20,000 \mathrm{rpm})$ of titanium dioxide, while a simple decantation process was used for zinc oxide. The particles obtained were dried in the oven at a temperature of $333 \mathrm{~K}$ for $48 \mathrm{~h}$, scraped using polymer scapula, collected using bird's feather, and stored in the plastic tubes. UV-visible absorption spectra of the as synthesized colloidal solution and methanol dispersed heat treated samples were recorded using PerkinElemr Lambda-35 double beam spectrophotometer. Atomic force microscopy (AFM) and magnetic force microscopy (MFM) images of as synthesized zinc oxide and titanium dioxide samples were recorded using a high-resolution scanning probe microscope
(SPM) attached with a magnetic probe (SPM multimode, Veeco Instruments, Inc., USA). Photoluminescence (PL) spectra of the as obtained zinc oxide and air annealed titanium dioxide samples were recorded using a tunable Xe-lamp source for the excitation and Edinburgh Instruments combined with FLSP920 steady spectrometer for the recording of emission spectra. XRD measurements were performed using $\mathrm{Cu}-\mathrm{K} \alpha$ $\left(\lambda=1.5402 \mathrm{~A}^{\circ}\right) \mathrm{X}$-ray line from Brucker-AXS D8 Advance $\mathrm{X}$-ray diffractometer, while transmission electron microscope (TEM) images were obtained using Technai G-20-stwin Transmission Electron Microscope operating at a $100 \mathrm{kV}$ accelerating potential with $1.44 \mathrm{~A}^{\circ}$ point and $2.32 \mathrm{~A}^{\circ}$ line resolution. The synthesis procedure involves only laser irradiation of high purity (99.99\%) zinc and titanium metal rods placed at the bottom of glass vessel containing high purity double distilled water. None of the three elements (target rods, water, and glass) used for particle synthesis are magnetic; therefore, contamination of samples by magnetic elements can be safely ignored.

\section{RESULTS}

X-ray diffraction spectrum of the as synthesized NPs illustrate that LA-PLA of Ti in water produces titanium dioxide NPs with abundance in anatase phase, which get transformed into lower ratio of anatase to rutile phases after annealing at $623 \mathrm{~K}$ for $9 \mathrm{~h}$ (Fig. 1(a)). XRD data shown in Fig. 1(b) exemplify that high quality zinc oxide NPs with hexagonal wurtzite crystal symmetry along with the traces of zinc and zinc hydroxide are produced by laser ablation of zinc in water. Results of Scherrer's formula for average sizes
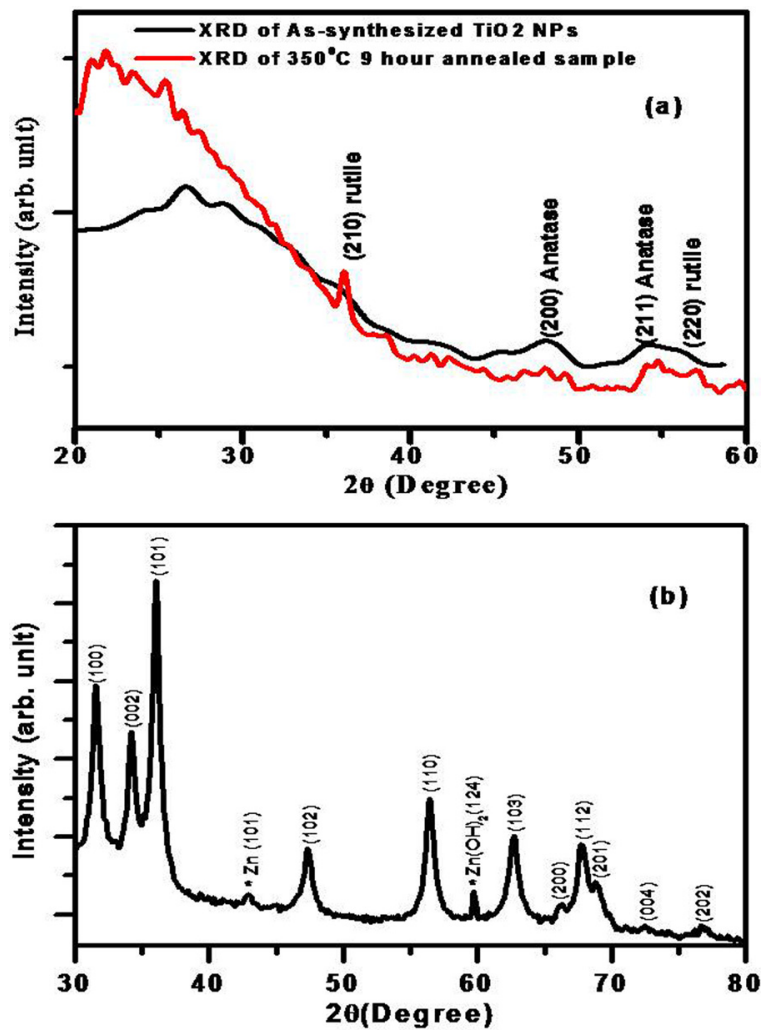

FIG. 1. X-ray diffraction spectra of (a) as synthesized and $623 \mathrm{~K}, 9 \mathrm{~h}$ annealed titanium dioxide NPs and (b) as synthesized zinc oxide NPs. 
of zinc oxide NPs, and anatase phases of as synthesized and $623 \mathrm{~K}, 9 \mathrm{~h}$ annealed titanium dioxide NPs are $9 \pm 1.2$, $4.2 \pm 0.5$, and $4.4 \pm 0.3 \mathrm{~nm}$, respectively, while it is $\sim 9.8 \mathrm{~nm}$ for the rutile phase of $\mathrm{TiO}_{2}$ particles in the annealed sample (Figs.1(a) and 1(b)). TEM data support XRD results for the sizes of titanium dioxide as well as zinc oxide (Figs. 2(a) and 2(b)) NPs. As far as crystallinity of the particles are concerned, XRD data of as synthesized and $623 \mathrm{~K}$ annealed titanium dioxide samples illustrate their amorphous nature (Figs. 1(a) and 1(b)); however, strong diffraction peaks for zinc oxide NPs demonstrate its higher degree of crystallinity. The amorphous nature of anatase titanium dioxide and crystallinity of zinc oxide NPs are also reflected by selective area electron diffraction (SAED) pattern of $\mathrm{TiO}_{2}$ (inset in Fig. 2(a)) and HRTEM image (inset in Fig. 2(b)) of $\mathrm{ZnO}$, respectively.

Fig. 3(a) exhibits room temperature $\mathrm{M}-\mathrm{H}$ curves for empty diamagnetic sample holder, $623 \mathrm{~K}, 9 \mathrm{~h}$ air annealed $\mathrm{TiO}_{2}$ NPs with holder and for $\mathrm{TiO}_{2}$ after subtracting holder's magnetic contribution. Hysteresis curve of amorphous anatase/rutile diphasic titanium dioxide NPs shows high order of intrinsic room temperature ferromagnetism with 42.17 memu/g saturation magnetization $\left(\mathrm{M}_{\mathrm{s}}\right), 5.78 \mathrm{memu} / \mathrm{g}$ magnetic retentivity $\left(\mathrm{M}_{\mathrm{r}}\right)$, and 102.95 Gauss magnetic coercivity $\left(\mathrm{H}_{\mathrm{C}}\right)$. Similar M-H curves for zinc oxide NPs having 62.37 and $2.04 \mathrm{memu} / \mathrm{g}$ respective values for $\mathrm{M}_{\mathrm{s}}$ and $\mathrm{M}_{\mathrm{r}}$ and 78.83 Gauss of $\mathrm{H}_{\mathrm{c}}$ are illustrated in Fig. 3(b). Both samples exhibit well defined hysteresis loops at $300 \mathrm{~K}$, which is the signature of robust high temperature real intrinsic ferromagnetic behavior. Comparative hysteresis curves for the as synthesized zinc oxide and $623 \mathrm{~K}, 9 \mathrm{~h}$ air annealed titanium dioxide NPs are presented in Fig. 3(c). Magnetization-temperature curves for zinc oxide and titanium dioxide NPs shown in Fig. 3(d) illustrate high values of Curie temperatures $\left(T_{C}\right)$ having $693 \mathrm{~K}$ for zinc oxide and more than $950 \mathrm{~K}$ for titanium dioxide NPs. The observed value of $\mathrm{M}_{\mathrm{s}}$ for zinc oxide NPs in the present communication is $1.25 \times 10^{2}$ times larger than that reported by Sundaresan et al. ${ }^{16}$ and almost 30 times higher than the results observed by Garcia et al. ${ }^{11}$ In addition to these, Sundaresan et al. used $673 \mathrm{~K}$ to anneal the samples (no magnetism in unannealed sample) for creating defects, while Garcia et al. used capping of $\mathrm{ZnO}$ particles by organic molecules to modify local electronic configuration responsible for RTF. Experimental studies of RTF for undoped titanium dioxide materials are mostly constrained into thin films. Saturation magnetization value for $\mathrm{TiO}_{2} \mathrm{NPs}$ in the present work is almost 4.5 fold larger than the only one report available until now for particles. ${ }^{17}$

Three dimensional AFM and MFM images of the as synthesized titanium dioxide and zinc oxide NPs are shown in Fig. 4. In AFM and MFM images, the color scale bar (in the right) from dark to light shows an increase in size and an increase in magnetic moment, respectively. AFM image of the as synthesized titanium dioxide sample (Fig. 4(a)) has some larger sized particles encircled by blue lines. Positions corresponding to larger sized particles in the MFM image (Fig. 4(b)) have larger magnetic moments, which are observed in the form of pinkish strips (encircled blue lines.) These strips are the signatures of strong ferromagnetic domains. The correlation between the AFM and MFM images of the as synthesized anatase titanium dioxide NPs shows that larger sized NPs have higher magnetic moments. In addition to these strong ferromagnetic domains, the MFM image (Fig. 4(b)) clearly shows light and dark strips (dark strip is highlighted by double headed blue arrows) corresponding to the weak ferromagnetic and non-magnetic domains, respectively. It is evident from the MFM image of the as synthesized $\mathrm{TiO}_{2}$ NPs that ferromagnetic centers in titanium dioxide NPs hold ferromagnetism for long range $(\approx 500 \mathrm{~nm})$ of distances. In contrast to the size dependent magnetism results of $\mathrm{TiO}_{2} \mathrm{NPs}$, smaller size zinc oxide NPs have larger degree of ferromagnetism. In the AFM image (Fig. 4(c)) of zinc oxide, there are some larger sized particles as shown by blue arrows, but at the corresponding positions in the MFM, there are almost no or very small magnetic domains. The smaller sized particles encircled by blue dotted lines (Fig. 4(c)) correspond for higher order of magnetic moments (Fig. 4(d)) as evidenced by clear observation of large number of ferromagnetic domains dispersed inside the encircled region. Larger density of magnetic particles on the grid (Fig. 4(a)) causes interaction between their magnetic moments and formation of strip shaped magnetic domains (Fig. 4(b)), while there are no such magnetic domains in the MFM of zinc oxide (Fig. 4(d)) due to comparatively larger distances between neighboring magnetic elements (Fig. $4(c))$.

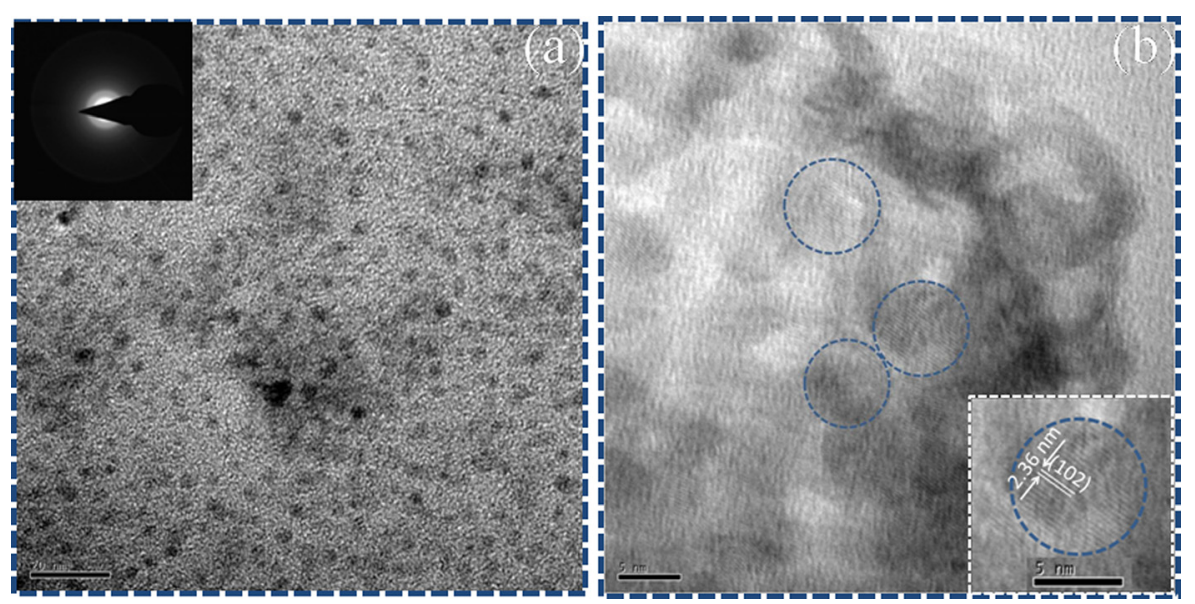

FIG. 2. TEM images of as synthesized (a) titanium dioxide and (b) zinc oxide NPs. 

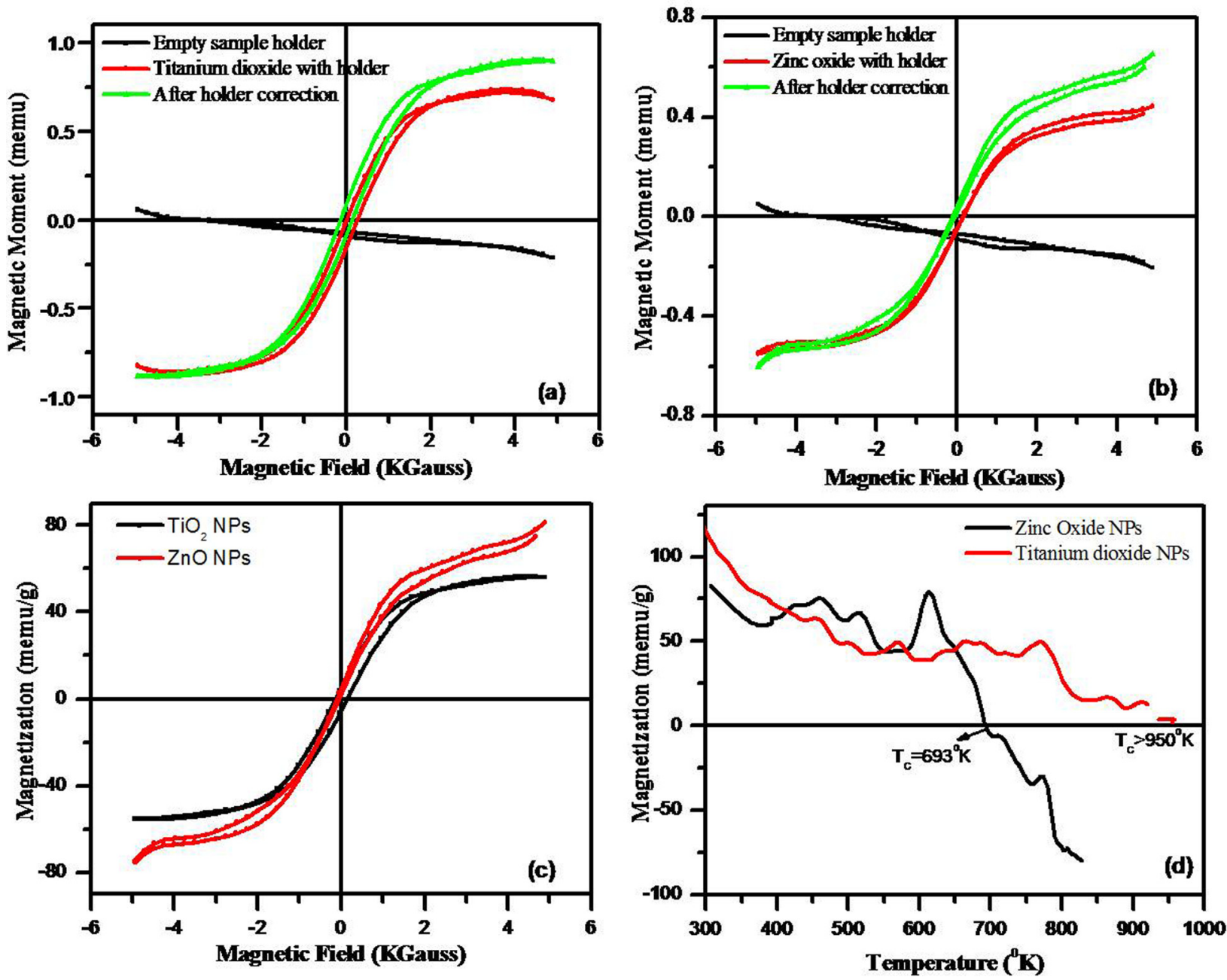

FIG. 3. M-H curve for (a) $\mathrm{TiO}_{2}$ nanoparticles; empty sample holder, sample filled in the sample holder, and after holder subtraction; (b) zinc oxide nanoparticles; empty sample holder, sample filled in the sample holder, and after holder subtraction; (c) comparative M-H curve for zinc oxide and titanium dioxide nanoparticles; and (d) comparative temperature-magnetization curve for zinc oxide and titanium dioxide nanoparticles.
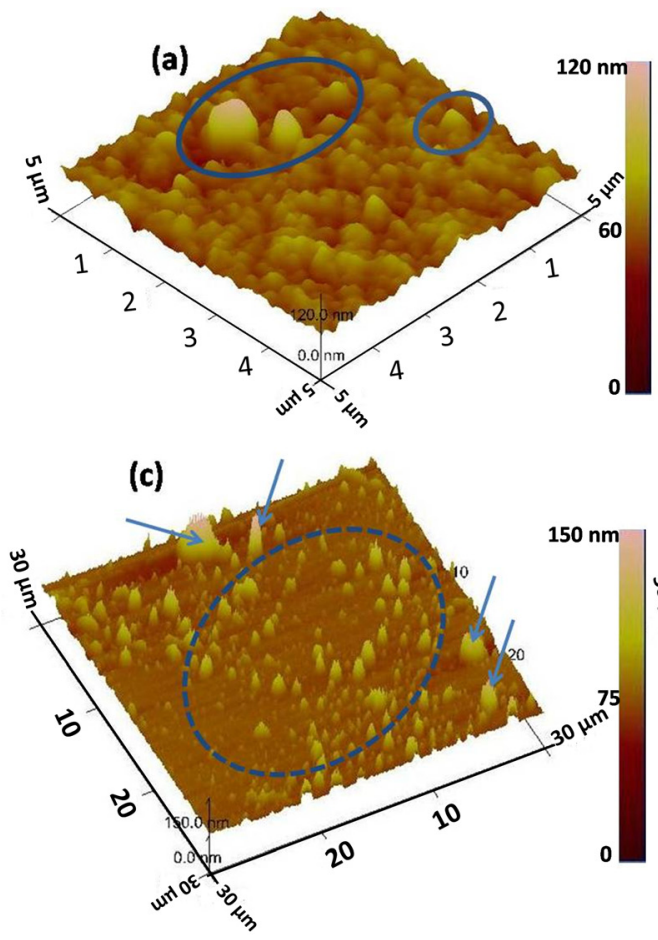

(b)

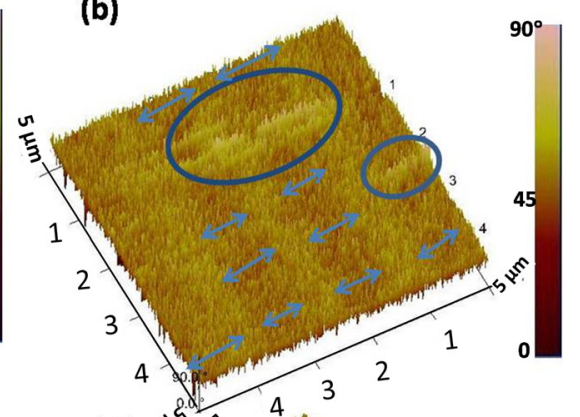

(d)

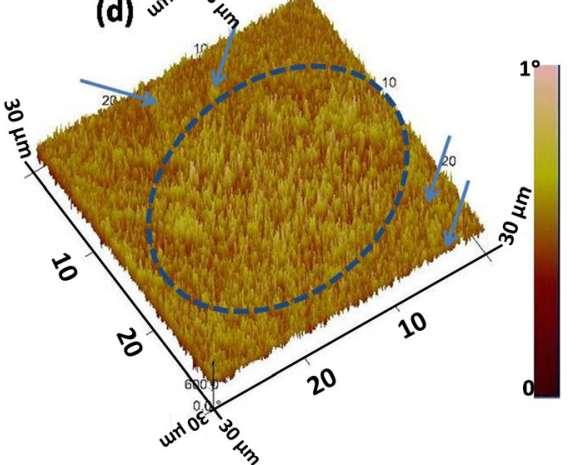

FIG. 4. (a) AFM and (b) corresponding MFM images of as-synthesized $\mathrm{TiO}_{2}$ NPs and (c) AFM and (d) corresponding MFM images of as-synthesized ZnO NPs. 

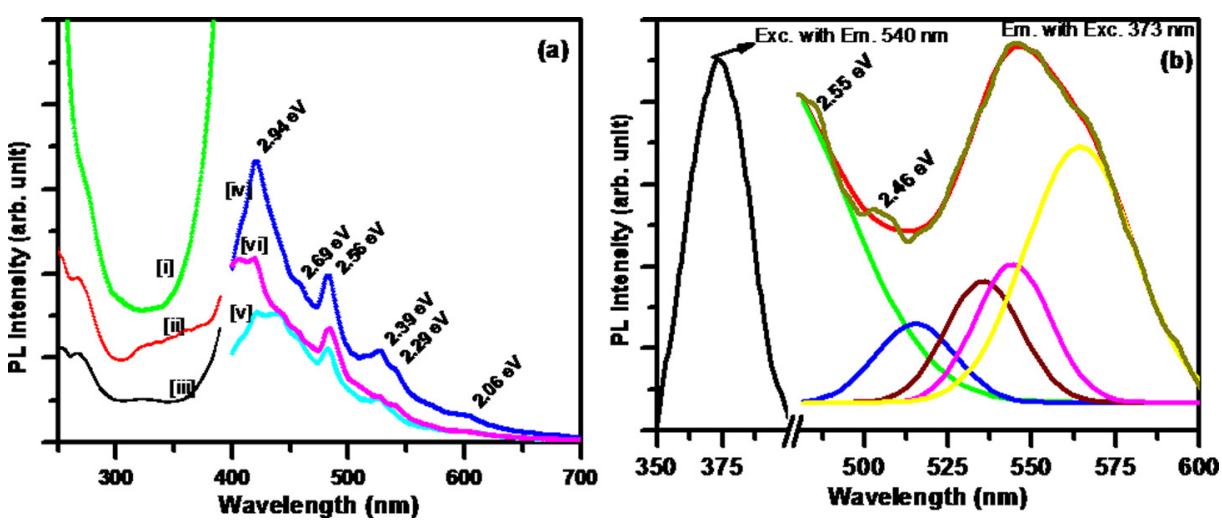

FIG. 5. Excitation and PL spectra from (a) titanium dioxide and (b) zinc oxide NPs. (In (a), numbers [i], [ii], and [iii] illustrate excitation spectra with emission at 526, 482, and $420 \mathrm{~nm}$ wavelength, respectively, while numbers [iv], [v], and [vi] illustrate emission spectra with $\lambda_{\text {exc. }}=269,320$, and $360 \mathrm{~nm}$, respectively.)
Excitation and PL spectra of $623 \mathrm{~K}, 9 \mathrm{~h}$ air annealed $\mathrm{TiO}_{2}$ NPs (Bandgap, $\mathrm{E}_{\mathrm{g}}=4.5 \mathrm{eV}$ ) are illustrated in Fig. 5(a), which are very similar in the PL structures observed in the previous reports. ${ }^{19,20}$ Five emission peaks with photon energies $2.94,2.69,2.56,2.39$, and $2.06 \mathrm{eVs}$ are observed, corresponding to all the three excitations $\left(E_{\text {exc. }}\right)$ of $4.6,3.86$, and $3.44 \mathrm{eV}$ energies. Much smaller energies of emission peaks over those of band gap and excitation energies proves the presence of several defect electronic states in the band gap, serving as electron and hole trap states responsible for observed PL emissions and ferromagnetism. Titanium dioxide is a non-stoichiometric compound and is generally known as oxygen deficient $\left(\mathrm{TiO}_{2-\delta}\right)$ material; therefore, predominant defects in titanium dioxide are oxygen vacancies. Strong oxidation of titanium dioxide under elevated temperature originates $\mathrm{Ti}$ ion vacancy, which acts as a hole trap level. Since PL peak at $2.94 \mathrm{eV}$ is most intense and also appears with the excitation of $3.44 \mathrm{eV}$ $\left(<\mathrm{E}_{\mathrm{g}}=4.5 \mathrm{eV}\right)$, it may be associated with the transition between oxygen vacancy $\left(\mathrm{V}_{\mathrm{O}}\right)$ and neutral/single charged $\mathrm{Ti}$ vacancy $\left(V_{T i}^{*} / V_{T i}^{\times}\right)$having energy levels $1.18 \mathrm{eV}$ below the bottom of conduction band and $0.38 \mathrm{eV}$ above the top of valance band, respectively. The appearance of these emission bands shows that oxygen vacancies $\left(V_{O}^{X}, V_{O}^{*}\right.$, and $\left.V_{O}^{* *}\right)$, titanium interstitials $\left(T i_{i}^{X}, T i_{i}^{*}, T i_{i}^{* *}\right.$, and $\left.T i_{i}^{* *}\right)$, and titanium vacancies $\left(V_{T i}^{X}, V_{T i}^{*}, V_{T i}^{\cdots}\right.$, and $\left.V_{T i}^{\cdots *}\right)$ lattice defects are present in the titanium dioxide NPs (Fig. 6(a)). Weak emission at $2.06 \mathrm{eV}$ may be due to the presence of rutile phase in the trace. ${ }^{21}$

Neutral oxygen vacancy in the $\mathrm{TiO}_{2}$ NPs causes lattice distortion and induces ferromagnetic states with magnetic moments of 0.22 and $0.06 \mu_{\mathrm{B}}$ on every neighboring Ti atoms in rutile and anatase titanium dioxide NPs, respectively. ${ }^{22}$ Neutral $\left(V_{T i}^{\times}\right)$and double ionized $\left(V_{T i}^{* *}\right)$ titanium vacancies produce holes in the narrow non-bonding oxygen $2 \mathrm{p} \pi$ bonds and originate collective magnetic moments of 3.5 and $2.0 \mu_{\mathrm{B}}$, respectively, in undoped anatase titanium dioxide NPs. ${ }^{23} \mathrm{On}$ the other hand, $\mathrm{Ti}^{+3}$ ions on the substitutional sites accompanied by oxygen vacancies having one unpaired electron can also induce local magnetic moment. ${ }^{17}$ As we have PL peaks corresponding to oxygen and titanium vacancies, these point defects may be responsible for observed ferromagnetism in undoped titanium dioxide NPs in the case of present investigation.

PL and excitation spectra of zinc oxide NPs, illustrated in Fig. 5(b), have a single intense excitation peak $(E m .=540 \mathrm{~nm})$ centered at $373 \mathrm{~nm}$ that corresponds to band gap absorption, while emission spectrum corresponding to $373 \mathrm{~nm}$ excitation has a broad green-yellow band in the spectral region of $510-610 \mathrm{~nm}$. The observed broad band PL peak is deconvoluted into three Gaussian bands centered at 535, 544, and $564 \mathrm{~nm}$. It is widely accepted that the green $(\sim 510-550 \mathrm{~nm})$ and yellow $(\sim 550-610 \mathrm{~nm})$ emission bands commonly originate from single $\left(V_{O}^{+}\right)$and doubly $\left(V_{O}^{++}\right)$charged oxygen vacancies, ${ }^{24,25}$ respectively. Shoulders at $480 \mathrm{~nm}(2.58 \mathrm{eV})$ and $505 \mathrm{~nm}(2.45 \mathrm{eV})$ may be associated with neutral/single/double charged zinc interstitials $\left(Z n_{i}^{X}, Z n_{i}^{*}\right.$, and $\left.Z n_{i}^{* *}\right)$ and zinc vacancies $\left(V_{Z n}^{\cdot} / V_{Z n}^{X}\right)$, respectively (Fig. 6(b)). Single ionized oxygen vacancy $\left(V_{O}^{+}\right)$is the dominant defect, but zinc interstitials and zinc vacancies are also present in the zinc oxide NPs sample. Single ionized oxygen and neutral zinc vacancies in zinc oxide NPs may induce magnetic moments on their neighboring ions.


FIG. 6. Energy level diagram of the various defect levels in the bandgap responsible for various emissions from LP-PLA produced (a) titanium dioxide and (b) zinc oxide nanoparticles. 


\section{DISCUSSION}

Here, it is observed that the magnitude of ferromagnetism in titanium dioxide increases with the size of particles, while it shows inverse relationship with the size for zinc oxide NPs. This result predicts that ferromagnetism in zinc oxide depends on the surface area, while for titanium dioxide, it has volume dependence. In other words, one can say that the defect responsible for ferromagnetism in the case of zinc oxide seems to be surface preferable, while for titanium dioxide, it may prefer to reside inside the volume. Golightly and Castleman reported that oxygen vacancy defect concentration is higher in larger sized LA-PLA produced $\mathrm{TiO}_{2-\delta}$ NPs and for a single spherical NP, oxygen vacancy concentration increases from the surface to the centre. ${ }^{26}$ Larger volumes in bigger sized $\mathrm{TiO}_{2-\delta}$ NPs correspond for higher density of oxygen vacancies, and each vacancy in volume corresponds to a larger number of neighboring $\mathrm{Ti}$ atoms as compared to those on the surface, which results for higher degree of ferromagnetism into larger sized $\mathrm{TiO}_{2-\delta}$ NPs. Additionally, oxygen vacancies inside the volume may get substituted by $\mathrm{Ti}^{+3}$ ions with one unpaired $3 \mathrm{~d}$ electrons that induce local magnetic moments on neighboring $\mathrm{Ti}$ atoms. Since defects inside the bulk have larger number of neighboring $\mathrm{Ti}$ atoms as compared to those on the surface, higher ferromagnetism for larger sized particles of $\mathrm{TiO}_{2}$ may be the consequence of a similar phenomenon. It is reported that neutral zinc vacancy, preferable to reside on the surface, introduces spin polarization on the top of valance band that results ferromagnetism in zinc oxide thin films. ${ }^{27}$ The absence of $3 d$ electron in zinc atoms makes unpairing of $2 p$ electrons of $\mathrm{O}$ atoms in the immediate vicinity of zinc vacancies. It is not only easier to introduce neutral zinc vacancies in $\mathrm{ZnO}$ nanowires (2D confinement) compared to $\mathrm{ZnO}$ thin film (1D confinement), but also resulting higher magnetic moment, ${ }^{27}$ which suggests that $\mathrm{ZnO}$ spherical NPs (3D confinement) should have easier generation of zinc vacancies and higher magnetic moment than zinc oxide nanowires and thin films. High temperature $(\approx 6000 \mathrm{~K})$ and pressure $(1 \mathrm{GP})$ of water confined laser produced plasma may have chances to create neutral zinc vacancies and other point defects responsible for PL emission from violet to red optical regions and related $\mathrm{RTF}^{28}$ Surface residing nature, easiest to introduce in the case of spherical particles, highest resulting magnetic moment of zinc neutral vacancies in zinc oxide NPs, non-equilibrium conditions of LP-PLA for the synthesis at high temperature and pressure, and PL emission at $2.45 \mathrm{eV}$ suggest the presence of a significant amount of zinc neutral vacancies as defect centers on the surface of zinc oxide particles in the present investigation.

Laser ablation of metal target in active liquid media is a highly non-equilibrium processing, which allows synthesis of novel phases of materials with plenty of defect centers. Scheme for the synthesis is demonstrated in Fig. 7. Laser beam irradiates metal target at target-liquid interface and creates plasma plume, which supersonically expands perpendicular to the target surface under the water confinement. Water layer in front of the expanding plasma plume and shock wave generated by plasma liquid interaction exerts extra pressure on the plasma surface, which results in high temperature $\left(\sim 10^{3} \mathrm{~K}\right)$ and pressure $(\mathrm{GPa})$ inside the plasma plume. Expanding plasma plume under highly confined environment ionizes water molecules at plasma-water interface, which may be termed as plasma induced plasma. Laser produced plasma have ionized species from target materials, while plasma induced plasma consists of ionized species from water molecules. Cationic species such as $\mathrm{Ti}^{+4}$ or $\mathrm{Zn}^{++}$from laser produced plasma react with the anionic species such as $\mathrm{O}^{-}$or $\mathrm{OH}^{-}$from plasma induced plasma at plasma liquid interface to generate oxide/hydroxide molecules under highly non-equilibrium environment. The as produced molecules get rapidly quenched into particles and may have the possibility of having some un-reacted cations/ anions/neutral atoms or their vacancies into the lattice. Excess or deficiency of the target or water species into NPs acts as defect centers and may be responsible for the observed RTF in the present investigation.

\section{CONCLUSION AND FUTURE PROSPECTS}

Summarizing our results, we conclude that (a) zinc oxide and titanium dioxide ferromagnetic quantum dots with high Curie temperatures are synthesized by purely green and non-equilibrium LP-PLA method. (b) The degree of ferromagnetism is higher for larger sized titanium dioxide particles $\left(M \alpha R^{n} ; n \geq 1\right)$, while it is reverse for zinc oxide ( $M \alpha 1 /$ $\mathrm{R}^{\mathrm{m}} ; \mathrm{m} \geq 1$ ) NPs. (c) Point defects such as single ionized oxygen and neutral zinc vacancies in zinc oxide, while neutral oxygen and $\mathrm{Ti}$ vacancies and substitutional $\mathrm{Ti}^{+3}$ ions on oxygen vacancy site for titanium dioxide NPs may be the predominant defect centers responsible for RTF. Determination of values of $\mathrm{m}$ and $\mathrm{n}$ by measuring $\mathrm{M}-\mathrm{H}$ curves of zinc oxide

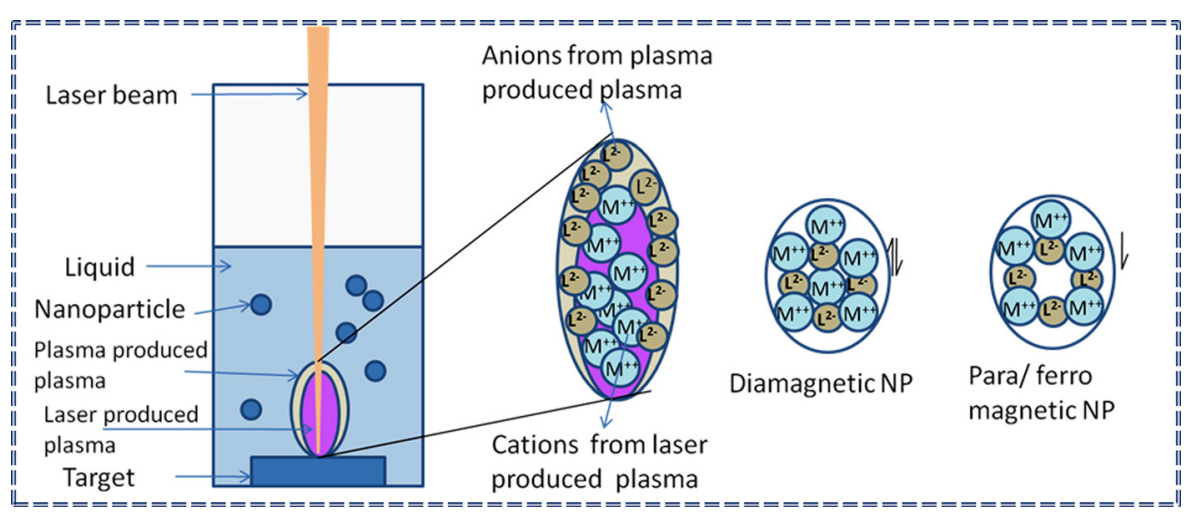

FIG. 7. Scheme of LA-PLA for particle synthesis with demonstration of diamagnetic and para/ferromagnetic NPs. 
and titanium dioxide NPs of different sizes is our proposed future plan. Results of our experimental investigation reveal that defect inside the nonmagnetic semiconductor materials is an effective alternate of transition metals: semiconductor magnetic systems for room/higher temperature DMSs. Such materials are highly important for biological applications where transition metals have adverse effects.

\section{ACKNOWLEDGMENTS}

Dr. S. C. Singh is thankful to Irish Research Council for Science, Engineering, and Technology (now called the Irish Research Council) for an EMPOWER postdoctoral fellowship to work at National Centre for Plasma Science and Technology. We are also thankful to DST-SERB, Grant No. SR/FTP/PS-106/2012 for current financial assistance. The authors are thankful to Samanta and Santa Chawala both from National Physical Laboratory, New Delhi, for AFM/ MFM and PL measurements, respectively. Prof. B. R. Mehta, IIT New Delhi is also acknowledged for TEM measurements.

${ }^{1}$ H. Ohno, Science 281, 951 (1998).

${ }^{2}$ P. Sharma, A. Gupta, K. V. Rao, F. J. Owens, R. Sharma, R. Ahuja, J. M. O. Guillen, B. Johansson, and G. A. Gehring, Nat. Mater. 2, 673 (2003).

${ }^{3}$ J. M. D. Coey, M. Venkatesan, and C. B. Fitzgerald, Nat. Mater. 4, 173 (2005).

${ }^{4}$ X. Wang, J. Xu, B. Zhang, H. Yu, J. Wang, X. Zhang, J. Yu, and Q. Li, Adv. Mater. 18, 2476 (2006).

${ }^{5}$ K. Ueda, H. Tabata, and T. Kawai, Appl. Phys. Lett. 79, 988 (2001); S. Ghoshal and P. S. A. Kumar, J. Phys.: Condens. Matter 20, 192201 (2008); R. P. Borges, R. C. da Silva, S. Magalhaes, M. M. Cruz, and M. Godinho, J. Phys.: Condens. Matter 19, 476207 (2007); S. Banerjee, M. Mandal, N. Gayathri, and M. Sardar, Appl. Phys. Lett. 91, 182501 (2007).

${ }^{6}$ Y. Matsumoto, M. Murakami, T. Shono, T. Hasegawa, T. Fukumura, M. Kawasaki, P. Ahmet, T. Chikyow, S. Koshihara, and H. Koinuma, Science 291, 854 (2001).

${ }^{7}$ L. Sangaletti, M. C. Mozzati, P. Galinetto, C. B. Azzoni, A. Speghini, M. Bettinelli, and G. Calestani, J. Phys.: Condens. Matter 18, 7643 (2006).

${ }^{8}$ H. Pan, J. B. Yi, L. Shen, R. Q. Wu, J. H. Yang, J. Y. Lin, Y. P. Feng, J. Ding, L. H. Van, and J. H. Yin, Phys. Rev. Lett. 99, 127201 (2007).
${ }^{9}$ S. Chawla, K. Jayanthi, and R. K. Kotnala, Phys. Rev. B 79, 125204 (2009).

${ }^{10}$ J. B. Yi, C. C. Lim, G. Z. Xing, H. M. Fan, L. H. Van, S. L. Huang, K. S. Yang, X. L. Huang, X. B. Qin, B. Y. Wang, T. Wu, L. Wang, H. T. Zhang, X. Y. Gao, T. Liu, A. T. S. Wee, Y. P. Feng, and J. Ding, Phys. Rev. Lett. 104, 137201 (2010).

${ }^{11}$ M. A. Garcia, J. M. Merino, E. Fernandez Pinel, A. Quesada, J. de la Venta, M. L. Ruız Gonzalez, G. R. Castro, P. Crespo, J. Llopis, J. M. Gonzalez-Calbet, and A. Hernando, Nano Lett. 7, 1489 (2007).

${ }^{12}$ M. Venkatesan, C. B. Fitzgerald, and J. M. D. Coey, Nature (London) 430, 630 (2004).

${ }^{13}$ S. D. Yoon, Y. Chen, A. Yang, T. L. Goodrich, X. Zuo, D. A. Arena, K. Ziemer, C. Vittoria, and V. G. Harris, J. Phys.: Condens. Matter 18, L355 (2006).

${ }^{14}$ S. R. Shinde, S. B. Ogale, J. S. Higgins, H. Zheng, A. J. Millis, V. N. Kulkarni, R. Ramesh, R. L. Greene, and T. Venkatesan, Phys. Re. Lett. 92, 166601 (2004).

${ }^{15}$ N. H. Hong, J. Sakai, N. Poirot, and V. Brize, Phys. Rev. B 73, 132404 (2006).

${ }^{16}$ A. Sundaresan, R. Bhargavi, N. Rangarajan, U. Siddesh, and C. N. R. Rao, Phys. Rev. B 74, 161306 (2006).

${ }^{17}$ S. Zhou, E. Cizmar, K. Potzger, M. Krause, G. Talut, M. Helm, J. Fassbender, S. A. Zvyagin, J. Wosnitza, and H. Schmidt, Phys. Rev. B 79, 113201 (2009).

${ }^{18}$ S. C. Singh and R. Gopal, J. Phys. Chem. C 114, 9277 (2010); S. C. Singh, R. K. Swarnkar, and R. Gopal, J. Nanosci. Nanotechnol. 9, 5367 (2009); S. C. Singh and R. Gopal, J. Phys. Chem. C 112, 2812 (2008); S. C. Singh, J. Nanopart. Res. 13, 4143 (2011).

${ }^{19}$ N. Serpone, D. Lawless, and R. Khairutdinov, J. Phys. Chem. 99, 16646 (1995).

${ }^{20}$ N. D. Abazovic, M. I. Comor, M. D. Dramicanin, D. J. Jovanovic, S. P. Ahrenkiel, and J. M. Nedeljkovic, J. Phys. Chem. B 110, 25366 (2006).

${ }^{21}$ J. Shi, J. Chen, Z. Feng, T. Chen, Y. Lian, X. Wang, and C. Li, J. Phys. Chem. C 111, 693 (2007).

${ }^{22}$ D. Kim, J. Hong, Y. R. Park, and K. J. Kim, J. Phys.: Condens. Matter 21, 195405 (2009).

${ }^{23}$ H. Peng, J. Li, S. S. Li, and J. B. Xia, Phys. Rev. B 79, 092411 (2009).

${ }^{24}$ K. Vasudevan, C. H. Seager, W. L. Warren, D. R. Tallant, and J. A. Voigt, Appl. Phys. Lett. 68, 403 (1996).

${ }^{25}$ A. van Dijken, E. A. Meulenkamp, D. Vanmaekelbergh, and A. Meijerink, J. Lumin. 87, 454 (2000).

${ }^{26}$ J. S. Golightly and A. W. Castleman, J. Phys. Chem. B 110, 19979 (2006).

${ }^{27}$ Q. Wang, Q. Sun, G. Chen, Y. Kawazoe, and P. Jena, Phys. Rev. B 77, 205411 (2008).

${ }^{28}$ H. Zeng, G. Duan, Y. Li, S. Yang, X. Xu, and W. Cai, Adv. Funct. Mater. 20, 561 (2010); J. Song, S. A. Kulinich, J. Li, Y. Liu, and H. Zeng, Angew. Chem. Int. Ed. 54, 462 (2015); S. C. Singh and R. Gopal, Appl. Surf. Sci. 258, 2211 (2012). 